Article

\title{
Family Support for Medical Nutritional Therapy and Dietary Intake among Japanese with Type 2 Diabetes (JDDM 56)
}

Chika Horikawa ${ }^{1}$, Mariko Hatta ${ }^{2}$, Sakiko Yoshizawa Morikawa ${ }^{3}{ }^{\circ}$, Yasunaga Takeda ${ }^{2}$, Mizuki Takeuchi ${ }^{2}$, Kazuya Fujihara ${ }^{2}$, Noriko Kato ${ }^{4}$, Hiroki Yokoyamaa ${ }^{5}$, Yoshio Kurihara ${ }^{6}$, Koichi Iwasaki ${ }^{7}$, Shiro Tanaka ${ }^{8}$, Hiroshi Maegawa ${ }^{9}$ and Hirohito Sone ${ }^{2, *}$ (1)

1 Department of Health and Nutrition, University of Niigata Prefecture Faculty of Human Life Studies, 471 Ebigase, Higashi-ku, Niigata 950-8680, Japan; horikawa@unii.ac.jp

2 Department of Hematology, Endocrinology, and Metabolism, Niigata University Faculty of Medicine, 1-757 Asahimachi-dori, Chuoh-ku, Niigata 951-8510, Japan; marichocolate.coffee02@gmail.com (M.H.); mr2.yac@gmail.com (Y.T.); y.mizuki3929@gmail.com (M.T.); kafujihara-dm@umin.ac.jp (K.F.)

3 Department of Food-Nutritional Sciences, Tokushima Bunri University Faculty of Human Life Sciences, 180 Hoji, Nishihama, Yamashiro-cho, Tokushima, Tokushima 770-8514, Japan; smorikawa@tks.bunri-u.ac.jp

4 Kato Clinic of Internal Medicine, 201, 3-11-14 Takasago, Katsushika-ku, Tokyo 125-0054, Japan; norikokato.0131@icloud.com

5 Jiyugaoka Medical Clinic, 6-4-3, Nishirokujominami, Obihiro, Hokkaido 080-0016, Japan; dryokoyama@yokoyamanaika.com

6 Kurihara Clinic, 5-7-28, Atsubetsuchuosanjo, Sapporo Atsubetsu-ku, Hokkaido 004-0053, Japan; ykuri@yg7.so-net.ne.jp

7 Iwasaki Naika Clinic, 1-30-13, Minamiiwakunimachi, Iwakuni, Yamaguchi 740-0034, Japan; kiwasaki@themis.ocn.ne.jp

8 Department of Clinical Biostatistics, Graduate School of Medicine, Kyoto University, Yoshida-Konoe-cho, Sakyo-ku, Kyoto 606-8501, Japan; tanaka.shiro.8n@kyoto-u.ac.jp

9 Department of Medicine, Division of Diabetology, Endocrinology and nephrology, Shiga University of Medical Science, Seta Tsukinowa-cho, Otsu, Sihga 520-2192, Japan; maegawa@belle.shiga-med.ac.jp

* Correspondence: sone@med.niigata-u.ac.jp; Tel.: +81-25-368-9024

Received: 11 August 2020; Accepted: 28 August 2020; Published: 31 August 2020

\begin{abstract}
The aim of this study was to investigate the association between habitual dietary intake for patients with diabetes and the content of family support for medical nutritional therapy (MNT). Analyzed were 289 Japanese with type 2 diabetes (men, 58.5\%; mean age, 62.0 years; mean HbA1c, $53.4 \mathrm{mmol} / \mathrm{mol}$ ) who completed the Food Frequency Questionnaire and Diabetes Family Behavior Checklist (DFBC). Relationships of mean values for food group intake to DFBC responses regarding MNT were examined using multivariate analysis of covariance. Positive response to "Praise for following diet" was associated with lower sweets intake (none: $60.1 \mathrm{~g} /$ day; $\geq$ once monthly: $50.9 \mathrm{~g} / \mathrm{day}$, $p=0.038$ ) and higher seasoning intake (none: $21.6 \mathrm{~g} /$ day, $\geq$ once monthly: $24.1 \mathrm{~g} / \mathrm{day}, p=0.046$ ). Energy intake was higher with positive responses to "Eat at the same time that you do" (none: $1636 \mathrm{kcal} / \mathrm{day}$, zonce monthly: $1818 \mathrm{kcal} /$ day, $p=0.038$ ). "Nags about not following diet" was associated with higher fish (none: $68.7 \mathrm{~g} /$ day, $\geq$ once monthly: $78.7 \mathrm{~g} /$ day, $p=0.042$ ) and salt intake (none: $8.3 \mathrm{~g} /$ day, zonce monthly: $9.0 \mathrm{~g} /$ day, $p=0.014$ ). Eating foods not part of the diabetic diet (none: $218.4 \mathrm{~g} /$ day, zonce monthly: $246.9 \mathrm{~g} / \mathrm{day}, p=0.014$ ) resulted in a higher vegetable intake. In females, significant differences in relationships in the overall analysis were reversed. Our results clarified relationships between types of family support of patients with type 2 diabetes and their dietary intake and the importance of sex differences for more effective MNT.
\end{abstract}


Keywords: type 2 diabetes mellitus; medical nutrition therapy; family support

\section{Introduction}

Self-care activities in the treatment of diabetes are crucial for improving glycemic control [1,2], reducing the risk of diabetes-related complications [2,3], and improving the prognosis [4]. However, performing ongoing self-care activities is sometimes difficult and adherence to medical nutrition therapy (MNT) has been shown to be poorer than adherence to medications [5,6], though MNT is an essential component of diabetes treatment.

Psychosocial care plays an important role in the practice of good self-care activities and has been associated with reducing stress, promoting positive emotions and behaviors $[7,8]$, improving adherence to recommended treatment regimens, improving glycemic control [8], and preventing the aggravation of the disease [9]. In psychosocial care, family support is the most easily accessible [10]. Our previous meta-analysis of interventional studies (follow-up period: 3.5-30 months) showed that $\mathrm{HbA1c}$ in diabetic patients was significantly lower when family members were involved than were not involved in family-oriented diabetes programs [11].

After completion of family-oriented programs for patients with diabetes, it was reported that self-efficacy in self-care activities improved, including adherence to MNT and adopting preferred dietary habits and intakes, such as more frequently selecting fat-reduced cooking methods such as baking or grilling, avoiding foods containing high amounts of fat and sugar, reducing fat intake, and increasing intakes of vegetables and fruits $[12,13]$. HbA1c levels were also shown to be lower in patients with type 2 diabetes who had family support for MNT, who shared education sessions for MNT, engaged in patient-family member communication about MNT, and received assistance with food preparation and grocery shopping [13-15].

Types of family support of diabetes patients have various and important effects on maintaining lifestyle changes and diabetes self-management. For example, it was reported that obstructive family behaviors with regard to patients' self-care efforts in diabetes care are barriers to effective self-efficacy and self-management adherence $[13,16,17]$. With respect to MNT, pressure and obstruction from family members regarding dietary matters were associated with lower dietary self-care adherence, higher diabetes-specific distress, and worsening blood glucose control among patients with type 2 diabetes [17-19]. However, evidence is sparse on the relationship between average dietary intake by food group and nutrient level and the content of family support regarding MNT in patients with diabetes. Without considering this relationship, it is difficult to determine whether such patients are receiving appropriate family support and to formulate effective family-oriented programs for MNT. Therefore, we investigated whether family support for MNT in Japanese outpatients with type 2 diabetes was related to average daily food and nutritional intake considering types of family support for MNT and patients' gender.

\section{Materials and Methods}

\subsection{Study Population}

Practicing diabetologists in eight diabetes clinics that were part of the Japan Diabetes Clinical Data Management Study Group (JDDM) participated in this cross-sectional study. Details of the JDDM have been described elsewhere [20,21]. Potential participants were 354 patients with type 2 diabetes aged $\geq 20$ years who underwent medical treatment in outpatient clinics from November 2015 to September 2018. The patients were requested to complete the Japanese version of the Diabetes Family Behavior Checklist (DFBC) [22,23] and the Food Frequency Questionnaire based on food groups (FFQg) [24]. Of these, 289 completed the questionnaires (collection rate, 81.6\%), and their data were analyzed. The protocol for the study, which is in accordance with the Declaration of Helsinki and the Ethical 
Guidelines for Clinical/Epidemiological Studies of the Japanese Ministry of Health Labor and Welfare, received ethical approval from the institutional review boards of all participating institutes and the ethics committee of the Niigata University Faculty of Medicine, Japan (No. 2317). Written informed consent was obtained from all participants.

\subsection{Family Support for MNT}

Family support for MNT was measured by the Japanese version of the DFBC. This instrument showed validity and reliability for the quick assessment of self-managed treatment behavior of Japanese type 2 diabetes patients [23]. It consists of "positive feedback" and "negative feedback" questions on five aspects of diabetes treatment: daily living, drug therapy, blood glucose measurement, exercise, and MNT. Patients answered questions by selecting "None," "Once a month," "Once a week," "Several times a week," or "At least once a day." The present study used diet regimen-specific subscales because previous studies using regimen-specific subscales such as diet and exercise reported that subscales were stronger indicators of their respective areas of regimen adherence than either overall positive or negative summary scores $[12,25,26]$. The diet regimen-specific subscale was used, which is composed of two positive and two negative items to assess family support specific to MNT. To elicit positive feedback, items were how often a particular family member would "praise you for following your diet" and "eat at the same time that you do." Negative feedback was determined by items on how often a family member "nagged you about not following your diet" and how often did family members "eat foods that are not part of your diabetes diet."

\subsection{Dietary Assessment}

Nutrition and food intakes were assessed by the FFQg. The FFQg is composed of items on 29 food groups and 10 kinds of cookery and elicits information on the average intake per week of each food or food group in commonly used units or portion sizes. After participants completed the questionnaire, a dietician reviewed the completed questionnaire with the participant. The FFQg was externally validated by comparison with weighed dietary records for seven continuous days of 66 individuals aged 19-60 years [24].

The correlation coefficients between the FFQg and 7-d dietary records for energy, protein, fat, and carbohydrate intakes were $0.47,0.42,0.39$, and 0.49 , respectively, and intakes of rice, bread, meat, fish, milk, dairy products, green-yellow vegetables, other vegetables, and fruits were $0.66,0.76$, $0.27,0.27,0.72,0.58,0.46,0.53$, and 0.64 , respectively. Intakes of 26 of the 31 nutrients and 22 of the 29 food groups were not significantly different between the two methods by paired $t$ tests. We used standardized software for population-based surveys and nutrition counseling in Japan (EIYO-KUN, manufactured at the site of the Shikoku University Nutrition Database) based on the Standard Tables of Food Composition in Japan [27] edited by the Japanese Ministry of Education, Culture, Sports, Science, and Technology to calculate nutrient and food intakes.

\subsection{Other Assessments}

$\mathrm{HbA1c}$ was measured in each clinic by high performance liquid chromatography. HbA1c assays were standardized by the Lab Test Committee of the Japan Diabetes Society (JDS) [28]. HbA1c values were converted from JDS values into National Glycohemoglobin Standardization Program (NGSP) equivalent values. NGSP equivalent values were calculated using the following formula: NGSP equivalent value $(\%)=$ JDS value $(\%)+0.416$.

Data on participants' age, sex, duration of diabetes, height, weight, body mass index (BMI), and use of oral hypoglycemic agents (OHA) and insulin were obtained by medical records from each diabetes clinic enrolled in this study. Participants were also asked whether he or she lived with a family member and who provided the strongest family support. 


\subsection{Statistical Analysis}

Categorical variables were expressed as numerals and percentages and were compared with Fisher's exact test or the $\chi^{2}$ test. Continuous variables were expressed as mean \pm SD and were compared using the $t$ test for comparisons in each group. Multiple regression analysis was used to assess the relation of energy, nutrients, and food intakes with the answer to the DFBC according to presence or absence of family support for MNT after accounting for potential confounders. We divided the answers of the diet regimen-specific subscale between none and greater or equal to once a month to investigate presence or absence of each type of family support for MNT. Energy intake was adjusted for age, sex, duration of diabetes, BMI, $\mathrm{HbA1c}$, using $\mathrm{OHA}$, and using insulin. Nutrients and food group intakes were adjusted for age, sex, duration of diabetes, BMI, HbA1c, using $\mathrm{OHA}$, using insulin, and energy intake per day. All analyses were performed by SPSS (version 23.0, IBM, New York, NY, USA), and statistical significance was considered for $p<0.05$.

\section{Results}

Table 1 shows the characteristics of the 289 type 2 diabetes patients. Participants' mean age was 62.0 years, mean duration of diabetes was 12.2 years, and $58.5 \%$ were men. Their mean $\mathrm{HbA} 1 \mathrm{c}$ value was $53.4 \mathrm{mmol} / \mathrm{mol}$ and mean BMI was $25.8 \mathrm{~kg} / \mathrm{m}^{2}$. An OHA and insulin were used by $46.4 \%$ and $14.2 \%$ of participants, respectively. There were no significant differences in age, diabetes duration, $\mathrm{HbA1c}$ level, BMI, and usage of an OHA and insulin between sexes.

As to family support for diabetes care, among male patients, $87.6 \%$ were mainly supported by wives, while $65.8 \%$ of female patients were mainly supported by husbands and $16.6 \%$ obtained the strongest family support from their children $(p<0.001)$. Most patients lived with their family regardless of sex (men, $93.5 \%$; women, $92.5 \%, p=0.816$ ). As for positive feedback on the DFBC scale for MNT, no significant difference was observed between participants' sex, and the rates of responses of $\geq$ once a month to "praise you for following your diet" and "eat at the same time that you do" were $46.4 \%$ and $82.7 \%$, respectively. Regarding negative feedback, men had a significantly higher rate of responses of $\geq$ once a month to "nag you about not following your diet" than women $(55.0 \%$ and $29.2 \%, p<0.001)$, but there was no significant difference in the responses to $\geq$ once a month to "eat foods that are not part of your diabetes diet" $(41.4 \%$ and $50.0 \%, p=0.339$ ).

The associations between dietary intake and responses to the DFBC scale for MNT are shown in Table 2. After adjusting for confounders, protein intake and proportion of protein as the percentage of energy supply were significantly greater in patients who received positive family support (i.e., praise for following diet) compared with those who did not. However, the differences were small clinically. Lower intake of sweets and higher intake of seasonings were observed in those who indicated praise for the following food than in those indicating "none" (sweets: none $60.1 \mathrm{~g} / \mathrm{day}$, $\geq$ once a month $50.9 \mathrm{~g} /$ day, $p=0.038$; and seasonings: none $21.6 \mathrm{~g} /$ day, $\geq$ once a month $24.1 \mathrm{~g} / \mathrm{day}, p=0.046)$. There were no significant associations between the responses to this item and other nutrient and food group intakes. Energy intake was significantly higher among those with a positive response to "eat at the same time that you do" than with a negative response (none $1636 \mathrm{kcal} / \mathrm{day}, \geq 1818 \mathrm{kcal} / \mathrm{day}, p=0.038$ ). There were no significant differences in nutrient and food group intakes according to responses to this item. In relation to negative family support, selecting "nag about not following diet" was significantly associated with higher fish intake (none $68.7 \mathrm{~g} /$ day; $\geq$ once a month $78.7 \mathrm{~g} / \mathrm{day}, p=0.042$ ) and high salt and sugar intakes (salt, none $8.3 \mathrm{~g} /$ day; $\geq$ once a month $9.0 \mathrm{~g} /$ day, $p=0.014$; sugar, none $6.5 \mathrm{~g} /$ day; $\geq$ once a month $8.3 \mathrm{~g} / \mathrm{day}, p=0.004)$. However, the difference in sugar intake was small in the daily diet. Those indicating "eat foods not part of the diabetes diet" had a higher fiber intake (none $12.5 \mathrm{~g} /$ day, $\geq$ once a month $13.5 \mathrm{~g} / \mathrm{day}, p=0.008$ ) and total vegetable intake (including "other vegetables" category) than those who answered "none" (total vegetables: none: $218.4 \mathrm{~g} /$ day, $\geq$ once a month $246.9 \mathrm{~g} /$ day, $p=0.014$, other vegetables intake: none $140.0 \mathrm{~g} /$ day, $\geq$ once a month $160.1 \mathrm{~g} /$ day, $p=0.004$ ).

Table 3 shows results of the subgroup analysis of men with type 2 diabetes according to dietary intake and responses to the DFBC scale for MNT. There were no notable differences in intake of 
nutrients and food groups according to baseline characteristics regardless of the response to "praise for following your diet." There was a significant difference only in energy intake among men who responded positively to "eat at the same time that you do" (none $1602 \mathrm{kcal} / \mathrm{day}$, $\geq$ once a month: $1910 \mathrm{kcal} / \mathrm{day}, p=0.038$ ). As for responses to "nag you about not following your diet," significantly higher fish, seaweed, and salt intake and lower intake of beverages except for alcohol were shown (fish: none $66.6 \mathrm{~g} / \mathrm{day}$, $\geq$ once a month $80.7 \mathrm{~g} / \mathrm{day}, p=0.034$; seaweed: none $4.0 \mathrm{~g} / \mathrm{day}$, $\geq$ once a month $5.3 \mathrm{~g} / \mathrm{day}, p=0.027$; salt: none $8.3 \mathrm{~g} /$ day, $\geq$ once a month $9.2 \mathrm{~g} / \mathrm{day}, p=0.032$; beverages except alcohol: none $82.6 \mathrm{~g} / \mathrm{day}$, $\geq$ once a month or more $48.8 \mathrm{~g} / \mathrm{day}, p=0.004)$. In those indicating "eat foods not part of your diabetes diet," the intakes of fiber and milk/dairy products were significantly higher (fiber: none $11.8 \mathrm{~g} /$ day, $\geq$ once a month $12.8 \mathrm{~g} /$ day, $p=0.040 ;$ milk/dairy products: none $71.7 \mathrm{~g} /$ day, $\geq$ once a month $96.2 \mathrm{~g} /$ day, $p=0.015)$.

With regard to women with type 2 diabetes (Table 4), the relationships with dietary intake and the responses to "praise for following your diet," "eat at the same time that you do," and "eat foods that are not part of your diabetes diet" were not significant. A significant difference was only shown when the female patients selected "nag about not following your diet." This expression of negative family support was only associated with high sugar intake (none $7.4 \mathrm{~g} /$ day, $\geq$ once a month $9.6 \mathrm{~g} / \mathrm{day}$, $p=0.015)$, but this difference was small as part of the dietary intake.

Table 1. Characteristics of study participants.

\begin{tabular}{|c|c|c|c|c|c|c|c|c|c|c|c|c|c|c|}
\hline & \multicolumn{4}{|c|}{ Overall $(N=289)$} & \multicolumn{4}{|c|}{$\operatorname{Men}(N=169)$} & \multicolumn{4}{|c|}{ Women $(N=120)$} & \multirow[b]{2}{*}{$\mathbf{p}$} & \\
\hline & $\mathbf{N}$ & Mean & \pm & SD & $\mathbf{N}$ & Mean & \pm & SD & $\mathbf{N}$ & Mean & \pm & SD & & \\
\hline Age (year) & 289 & 62.0 & \pm & 11.3 & 169 & 61.9 & \pm & 10.8 & 120 & 62.2 & \pm & 12.1 & 0.837 & + \\
\hline Men, \% & 169 & 58.5 & $\%$ & & 169 & 100.0 & $\%$ & & 0 & 0.0 & $\%$ & & - & \\
\hline Living with family, $\%$ & 268 & 92.7 & $\%$ & & 157 & 93.5 & $\%$ & & 111 & 92.5 & $\%$ & & 0.816 & $\ddagger$ \\
\hline Supported mainly by husband, \% & 78 & 27.0 & $\%$ & & 0 & 0.0 & $\%$ & & 79 & 65.8 & $\%$ & & $<0.001$ & $\S$ \\
\hline Supported mainly by wife, $\%$ & 144 & 49.8 & $\%$ & & 148 & 87.6 & $\%$ & & 0 & 0.0 & $\%$ & & & \\
\hline $\begin{array}{l}\text { Supported mainly by their } \\
\text { children, } \%\end{array}$ & 29 & 17.2 & $\%$ & & 1 & 0.6 & $\%$ & & 28 & 16.6 & $\%$ & & & \\
\hline $\begin{array}{l}\text { Supported mainly by their } \\
\text { parents, } \%\end{array}$ & 26 & 15.4 & $\%$ & & 17 & 10.1 & $\%$ & & 9 & 5.3 & $\%$ & & & \\
\hline $\begin{array}{l}\text { Supported mainly by others not } \\
\text { above, } \%\end{array}$ & 12 & 7.1 & $\%$ & & 3 & 1.8 & $\%$ & & 4 & 2.4 & $\%$ & & & \\
\hline Duration of diabetes (year) & 289 & 12.2 & \pm & 7.3 & 169 & 12.4 & \pm & 0.6 & 120 & 11.9 & \pm & 0.7 & 0.547 & $\dagger$ \\
\hline Height $(\mathrm{cm})$ & 289 & 162.1 & \pm & 8.6 & 169 & 167.2 & \pm & 0.5 & 120 & 154.9 & \pm & 0.5 & $<0.001$ & $t$ \\
\hline Weight (kg) & 289 & 67.9 & \pm & 15.7 & 169 & 71.4 & \pm & 1.1 & 120 & 63.0 & \pm & 1.4 & $<0.001$ & $t$ \\
\hline BMI $\left(\mathrm{kg} / \mathrm{cm}^{2}\right)$ & 289 & 25.8 & \pm & 5.0 & 169 & 25.5 & \pm & 0.3 & 120 & 26.1 & \pm & 0.5 & 0.380 & $\dagger$ \\
\hline $\mathrm{HbA1c}(\mathrm{mmol} / \mathrm{mol})$ & 289 & 53.4 & \pm & 6.4 & 169 & 52.5 & \pm & 5.4 & 120 & 54.7 & \pm & 7.5 & 0.006 & $t$ \\
\hline Using OHA, \% & 134 & 46.4 & $\%$ & & 83 & 49.1 & $\%$ & & 51 & 42.5 & $\%$ & & 0.283 & $\ddagger$ \\
\hline Using insulin, \% & 41 & 14.2 & $\%$ & & 20 & 11.8 & $\%$ & & 21 & 17.5 & $\%$ & & 0.231 & $\ddagger$ \\
\hline \multicolumn{15}{|l|}{ DFBC scale for MNT } \\
\hline \multicolumn{15}{|l|}{ Positive feedback } \\
\hline \multicolumn{15}{|c|}{ - Praise you for following your diet } \\
\hline None & 155 & 53.6 & $\%$ & & 79 & 46.7 & $\%$ & & 76 & 63.3 & $\%$ & & 0.075 & $\S$ \\
\hline Once a month & 45 & 15.6 & $\%$ & & 31 & 18.3 & $\%$ & & 14 & 11.7 & $\%$ & & & \\
\hline Once a week & 34 & 11.8 & $\%$ & & 21 & 12.4 & $\%$ & & 13 & 10.8 & $\%$ & & & \\
\hline Several times a week & 44 & 15.2 & $\%$ & & 31 & 18.3 & $\%$ & & 13 & 10.8 & $\%$ & & & \\
\hline At least once a day & 11 & 3.8 & $\%$ & & 7 & 4.1 & $\%$ & & 4 & 3.3 & $\%$ & & & \\
\hline \multicolumn{15}{|c|}{ - Eat at the same time that you do } \\
\hline None & 50 & 17.3 & $\%$ & & 30 & 17.8 & $\%$ & & 20 & 16.7 & $\%$ & & 0.718 & $\S$ \\
\hline Once a month & 7 & 2.4 & $\%$ & & 5 & 3.0 & $\%$ & & 2 & 1.7 & $\%$ & & & \\
\hline Once a week & 6 & 2.1 & $\%$ & & 2 & 1.2 & $\%$ & & 4 & 3.3 & $\%$ & & & \\
\hline Several times a week & 41 & 14.2 & $\%$ & & 24 & 14.2 & $\%$ & & 17 & 14.2 & $\%$ & & & \\
\hline At least once a day & 185 & 64.0 & $\%$ & & 108 & 63.9 & $\%$ & & 77 & 64.2 & $\%$ & & & \\
\hline \multicolumn{15}{|l|}{ Negative feedback } \\
\hline \multicolumn{15}{|c|}{ - Nag you about following your diet } \\
\hline None & 161 & 55.7 & $\%$ & & 76 & 45.0 & $\%$ & & 85 & 70.8 & $\%$ & & $<0.001$ & $\S$ \\
\hline Once a month & 61 & 21.1 & $\%$ & & 44 & 26.0 & $\%$ & & 17 & 14.2 & $\%$ & & & \\
\hline Once a week & 32 & 11.1 & $\%$ & & 25 & 14.8 & $\%$ & & 7 & 5.8 & $\%$ & & & \\
\hline Several times a week & 26 & 9.0 & $\%$ & & 20 & 11.8 & $\%$ & & 6 & 5.0 & $\%$ & & & \\
\hline At least once a day & 9 & 3.1 & $\%$ & & 4 & 2.4 & $\%$ & & 5 & 4.2 & $\%$ & & & \\
\hline \multicolumn{15}{|c|}{ - Eat foods that are not part of your diabetic diet } \\
\hline None & 159 & 55 & $\%$ & & 99 & 58.6 & $\%$ & & 60 & 50.0 & $\%$ & & 0.339 & $\S$ \\
\hline Once a month & 21 & 7.3 & $\%$ & & 14 & 8.3 & $\%$ & & 7 & 5.8 & $\%$ & & & \\
\hline Once a week & 23 & 8.0 & $\%$ & & 13 & 7.7 & $\%$ & & 10 & 8.3 & $\%$ & & & \\
\hline Several times a week & 41 & 14.2 & $\%$ & & 22 & 13.0 & $\%$ & & 19 & 15.8 & $\%$ & & & \\
\hline At least once a day & 45 & 15.6 & $\%$ & & 21 & 12.4 & $\%$ & & 24 & 20.0 & $\%$ & & & \\
\hline
\end{tabular}

†. $\mathrm{t}$ test, ‡. Fisher's exact test, §. Chi-squared test. BMI, body mass index; DFBC, Diabetes Family Behavior Checklist; MNT, medical nutrition therapy; OHA, oral hypoglycemic agent. 
Table 2. Dietary intake and response to the Diabetes Family Behavior Checklist (DFBC) scale for MNT in patients with type 2 diabetes $(N=289)$.

\begin{tabular}{|c|c|c|c|c|c|c|c|c|c|c|c|c|c|c|c|c|c|c|c|c|}
\hline & \multicolumn{5}{|c|}{ Praise You for Following Your Diet } & \multicolumn{5}{|c|}{ Eat at the Same Time that You do } & \multicolumn{5}{|c|}{ Nag You about Following Your diet } & \multicolumn{5}{|c|}{$\begin{array}{l}\text { Eat Foods that are not Part of } \\
\text { Your Diabetic Diet }\end{array}$} \\
\hline & \multicolumn{2}{|r|}{ None } & \multicolumn{2}{|c|}{$\geq$ Once a Month } & \multirow[b]{2}{*}{$p$} & \multicolumn{2}{|r|}{ None } & \multicolumn{2}{|c|}{$\geq$ Once a Month } & \multirow[b]{2}{*}{$p$} & \multicolumn{2}{|r|}{ None } & \multicolumn{2}{|c|}{$\geq$ Once a Month } & \multirow[b]{2}{*}{$p$} & \multicolumn{2}{|r|}{ None } & \multicolumn{2}{|c|}{$\geq$ Once a Month } & \multirow[b]{2}{*}{$p$} \\
\hline & Mean & {$[95 \% \mathrm{CI}]$} & Mean & {$[95 \% \mathrm{CI}]$} & & Mean & {$[95 \% \mathrm{CI}]$} & Mean & {$[95 \% \mathrm{CI}]$} & & Mean & {$[95 \% \mathrm{CI}]$} & Mean & {$[95 \% \mathrm{CI}]$} & & Mean & {$[95 \% \mathrm{CI}]$} & Mean & {$[95 \% \mathrm{CI}]$} & \\
\hline No. participants & 155 & & 134 & & & 50 & & 239 & & & 161 & & 128 & & & 159 & & 130 & & \\
\hline Energy (kcal) & 1792 & [1712-1873] & 1780 & [1693-1867] & 0.839 & 1636 & [1498-1774] & 1818 & [1755-1881] & 0.019 * & 1745 & [1666-1823] & 1839 & [1751-1928] & 0.124 & 1769 & [1690-1848] & 1808 & [1721-1896] & 0.515 \\
\hline Protein (g) & 64.0 & {$[62.5-65.5]$} & 66.4 & {$[64.8-68]$} & $0.038 *$ & 64.0 & {$[61.3-66.6]$} & 65.3 & [64.1-66.5] & 0.352 & 64.6 & {$[63.1-66.1]$} & & {$[64.0-67.4]$} & & 64.9 & {$[63.5-66.4]$} & & & 0.772 \\
\hline 6 Energy) & 14.3 & {$[14-14$} & 14.8 & [14.5-15.2] & $0.027 *$ & 14.4 & [13.8-14.9] & 14.6 & {$[14.3-$} & 0.4 & 14 & {$[14.2$} & 14 & 14.3. & & & & & & 0.409 \\
\hline Fat (g) & 57.8 & [55.9-59.7] & 58.1 & {$[56-60.1]$} & 0.836 & 57.7 & [54.5-61] & 58.0 & {$[56.5-5$} & 0.906 & 57.6 & $5.8-$ & 58 & $56.2-6$ & 0.6 & 58.0 & {$[56.2$} & & {$[55.7$} & 0.866 \\
\hline Fat (\% Energy) & 28.6 & {$[27.7-29.5]$} & 29.3 & {$[28.3-30.2]$} & 0.290 & 28.7 & {$[27.2-30.2]$} & 29.0 & [28.3-29.6] & 0.772 & 28.8 & {$[27.9-29.6]$} & 29.1 & {$[28.1-30.0]$} & 0.658 & 28.8 & & 29.0 & & 0.785 \\
\hline Carbohydrate (g) & 238.3 & {$[232.8-243.7]$} & 236.0 & {$[230.2-241.9]$} & 0.590 & 238.5 & [229.0-248.0] & 237.0 & [232.7-241.3] & 0.769 & 238.8 & [233.5-244.2] & 235.2 & [229.2-241.2] & 0.385 & 236.0 & [230.7-241.3] & 238.7 & [232.8-244.6] & 0.511 \\
\hline Carbohydrate (\% Energy) & 57.1 & [56-58.2] & 55.9 & {$[54.7-57.0]$} & 0.128 & 56.9 & {$[55.0-58.8]$} & 56.5 & {$[55.6-57.3]$} & 0.663 & 56.7 & {$[55.7-57.8]$} & 56.3 & [55.1-57.5] & 0.584 & 56.7 & {$[55.7-57.8]$} & 56.3 & {$[55.2-57.5]$} & 0.628 \\
\hline Fiber (g) & 12.8 & [12.3-13.3] & 13.1 & {$[12.5-13.6]$} & 0.484 & 13.3 & [12.4-14.2] & 12.8 & [12.4-13.2] & 0.368 & 12.9 & [12.4-13.4] & 12.9 & [12.3-13.4] & 0.873 & 12.5 & {$[12-12.9]$} & 13.5 & [12.9-14.0] & 0.008 \\
\hline Salt (g) & 8.3 & {$[8.0-8.7]$} & 8.9 & {$[8.5-9.3]$} & 0.053 & 8.2 & {$[7.5-8.8]$} & 8.7 & {$[8.4-9.0]$} & 0.185 & 8.3 & [7.9-8.6] & 9.0 & [8.6-9.4] & 0.014 * & 8.5 & [8.1-8.9] & 8.7 & [8.3-9.1] & 0.462 \\
\hline Potassium (mg) & 2200 & [2129-2271] & 2244 & [2168-2321] & 0.408 & 2237 & [2113-2360] & 2217 & [2161-2273] & 0.776 & 2219 & [2149-2288] & 2223 & [2145-2301] & 0.937 & 2181 & [2112-2250] & 2269 & [2193-2346] & 0.097 \\
\hline Grain $(g)$ & 367.9 & [349.0-386.8] & 372.1 & [351.7-392.5] & 0.768 & 358.1 & [325.2-391.1] & 372.3 & [357.3-387.2] & 0.443 & 372.7 & [354.2-391.2] & 366.2 & [345.4-387.1] & 0.657 & 368.3 & [349.8-386.9] & 371.7 & [351.1-392.3] & 0.813 \\
\hline Potato (g) & 27.8 & [23.5-32] & 31.6 & {$[27.0-36.2]$} & 0.236 & 30.2 & {$[22.8-37.7]$} & 29.4 & {$[26.0-32.8]$} & 0.842 & 27.8 & [23.7-32] & 31.7 & {$[27.0-36.4]$} & 0.237 & 27.9 & {$[23.7-32.0]$} & 31.6 & {$[27.0-36.2]$} & 0.246 \\
\hline Vegetables (g) & 228.4 & [210.8-246] & 234.4 & [215.5-253.4] & 0.652 & 235.2 & [204.5-265.9] & 230.4 & [216.4-244.3] & 0.779 & 234.9 & [217.6-252.1] & 226.6 & [207.1-246] & 0.539 & 218.4 & [201.3-235.5] & 246.9 & [227.9-265.8] & 0.031 \\
\hline Green-yellow vegetables (g) & 82.0 & [75.0-89.1] & 82.3 & {$[74.7-89.9]$} & 0.959 & 82.4 & [70.1-94.7] & 82.1 & [76.5-87.7] & 0.965 & 83.9 & $\begin{array}{l}\text { [77-90.9] } \\
\text { [ }\end{array}$ & 79.9 & {$[72.1-87.7]$} & 0.455 & 78.4 & [71.5-85.3] & 86.8 & [79.1-94.4] & 0.114 \\
\hline Other ve & 146.4 & {$[134.7-158.1]$} & 152.2 & {$[139.5-164.8]$} & 0.517 & 152.8 & [132.4-173.2] & 148.3 & {$[139-157.5]$} & 0.693 & 150.9 & [139.5-162.4] & 146.7 & {$[133.8-159.6]$} & 0.637 & 140.0 & [128.7-151.4] & 160.1 & {$[147.5-172.7]$} & 0.023 \\
\hline Seaweed & 4.9 & {$[4.3-5.5]$} & 5.5 & {$[4.9-6.2]$} & 0.174 & 5.3 & {$[4.2-6.4]$} & 5.2 & {$[4.7-5.6]$} & 0.791 & 4.9 & {$[4.3-5.5]$} & 5.6 & {$[4.9-6.3]$} & 0.115 & 4.8 & {$[4.2-5$} & 5.6 & {$[4.9-6.3]$} & 0.108 \\
\hline & 61.0 & {$[55.0-6$} & 65.9 & [59.4-72.4] & 0.285 & 68.4 & [57.9-78.9] & 62.2 & & & & & & & 0.6 & & & & {$[57.1-$} & 0.879 \\
\hline Fish/ & 69.3 & {$[6$} & 77 & & & & & & & & & & & & & & {$[67$.} & & & 0.770 \\
\hline Meat $/ \mathbb{P}_{1}$ & 73.5 & {$[6$} & 70.0 & {$[6$} & & 67. & {$[55.2-7$} & & & & & {$[6$} & & & 0. & & & & & \\
\hline & & & 28 & & & & & & & & & & & & & & & & & \\
\hline airy products $(\mathrm{g})$ & 121.7 & {$[107.7-13$} & 132.9 & [117.9-148.0] & & 122.4 & {$[98.0-14$} & 127.8 & [116.8-138.9] & & 129 & {$[115.9$} & 123.5 & {$[108$.} & & 132.8 & & 119.7 & & 0.215 \\
\hline Fruits & 91.1 & {$[81.4-10$} & 86.0 & {$[75.5-96.4]$} & 0.489 & 93.9 & {$[76.9-1$} & 87.6 & & 0.510 & 83.2 & & 95.6 & & 0.0 & 84.8 & & 93.5 & & 0.2 \\
\hline & 60.1 & & 50.9 & & & 62.8 & & 54 & & & 57 & & 54 & & & 57.9 & & 53 & & 0.2 \\
\hline Alcoholic beverages (g) & 76.4 & {$[58.8-9$} & 68.4 & & 0.552 & 78.4 & {$[47.6-1$} & 71. & {$[57.5-\varepsilon$} & 0.6 & 68.1 & {$[50.8-$} & 78 & {$[59.0-8$} & 0.4 & 79.7 & & 64.1 & {$[45-83.3]$} & 0.242 \\
\hline Other beverages (g) & 51.9 & {$[37.6-6$} & 44.4 & {$[28.9-$} & 0.489 & 53.3 & {$[28.3-$} & 47.4 & {$[36.1-$} & 0.675 & 57.3 & {$[43.3-$} & 37.3 & {$[21.5-53]$} & 0.069 & 47.4 & [33.4-61.5] & 49.7 & [34.1-65.2] & 0.836 \\
\hline Sugar $(g)$ & 7.1 & {$[6.3-7.8]$} & 7.6 & {$[6.7-8.4]$} & 0.393 & 7.0 & {$[5.6-8.3]$} & 7.4 & {$[6.7-8$.} & 0.614 & 6.5 & {$[5.8-7.3]$} & 8.3 & {$[7.4-9.1]$} & $0.004 *$ & 7.0 & {$[6.2-7.7]$} & 7.7 & {$[6.9-8.6]$} & 0.189 \\
\hline Nuts (g) & 2.8 & {$[2.1-3.5]$} & 2.7 & {$[2.0-3.5]$} & 0.902 & 2.3 & {$[1.1-3.4]$} & 2.9 & {$[2.4-3.4]$} & 0.331 & 2.8 & {$[2.1-3.4]$} & 2.7 & {$[2.0-3.5]$} & 0.930 & 2.6 & {$[2.0-3.3]$} & 3.0 & {$[2.2-3.7]$} & 0.477 \\
\hline Oil (g) & 11.3 & {$[10.2-12.4]$} & 12.0 & [10.8-13.2] & 0.380 & 10.3 & {$[8.4-12.2]$} & 11.9 & {$[11-12.8]$} & 0.133 & 11.1 & [10.0-12.2] & 12.3 & [11.1-13.5] & 0.176 & 11.7 & {$[10.6-12.7]$} & 11.6 & [10.4-12.8] & 0.963 \\
\hline Seasonings (g) & 21.6 & {$[20-23.3]$} & 24.1 & [22.4-25.9] & $0.046 *$ & 20.8 & {$[17.9-23.7]$} & 23.2 & [21.9-24.5] & 0.138 & 22.1 & [20.4-23.7] & 23.7 & [21.9-25.5] & 0.196 & 23.0 & [21.4-24.6] & 22.5 & {$[20.7-24.3]$} & 0.698 \\
\hline
\end{tabular}

${ }^{*} p<0.05$. Energy intake was adjusted for age, sex, duration of diabetes, BMI, HbA1c, using OHA, and using insulin. Nutrients and food group intakes were adjusted for age, sex, duration of diabetes, BMI, HbA1c, using OHA, using insulin, and energy intake per day. BMI, body mass index; OHA, oral hypoglycemic agent. 
Table 3. Dietary intake and response to the Diabetes Family Behavior Checklist scale for medical nutritional therapy in men with type 2 diabetes $(N=169)$.

\begin{tabular}{|c|c|c|c|c|c|c|c|c|c|c|c|c|c|c|c|c|c|c|c|c|}
\hline & \multicolumn{5}{|c|}{ Praise You for Following Your Diet } & \multicolumn{5}{|c|}{ Eat at the Same Time that You Do } & \multicolumn{5}{|c|}{ Nag You about Following Your Diet } & \multicolumn{5}{|c|}{$\begin{array}{l}\text { Eat Foods that are not Part of } \\
\text { Your Diabetic Diet }\end{array}$} \\
\hline & \multicolumn{2}{|r|}{ None } & \multicolumn{2}{|c|}{$\geq$ Once a Month } & \multirow[b]{2}{*}{$p$} & \multicolumn{2}{|r|}{ None } & \multicolumn{2}{|c|}{$\geq$ Once a Month } & \multirow[b]{2}{*}{$p$} & \multicolumn{2}{|r|}{ None } & \multicolumn{2}{|c|}{$\geq$ Once a Month } & \multirow[b]{2}{*}{$p$} & \multicolumn{2}{|r|}{ None } & \multicolumn{2}{|c|}{$\geq$ Once a Month } & \multirow[b]{2}{*}{$p$} \\
\hline & Mean & {$[95 \% \mathrm{CI}]$} & Mean & {$[95 \% \mathrm{CI}]$} & & Mean & {$[95 \% \mathrm{CI}]$} & Mean & {$[95 \% \mathrm{CI}]$} & & Mean & {$[95 \% \mathrm{CI}]$} & Mean & {$[95 \% \mathrm{CI}]$} & & Mean & {$[95 \% \mathrm{CI}]$} & Mean & {$[95 \% \mathrm{CI}]$} & \\
\hline No. participants & 79 & & 90 & & & 30 & & 139 & & & 76 & & 93 & & & 99 & & 70 & & \\
\hline Energy (kcal) & 1849 & [1725-1973] & 1861 & [1745-1977] & 0.888 & 1602 & [1407-1797] & 1910 & [1820-2000] & 0.005 * & 1821 & [1696-1946] & 1884 & [1771-1997] & 0.466 & 1824 & [1712-1936] & 1900 & [1766-2034] & 0.400 \\
\hline Protein $(\mathrm{g})$ & 64.5 & {$[62.3-66.8]$} & 67.3 & [65.2-69.5] & 0.080 & 63.3 & {$[59.5-67.0]$} & 66.6 & [64.9-68.3] & 0.109 & 65.0 & {$[62.7-67.3]$} & 66.9 & [64.8-68.9] & 0.246 & 65.9 & [63.9-68] & 66.2 & {$[63.7-68.7]$} & 0.875 \\
\hline Protein (\% Energy) & 13.9 & {$[13.5-14.4]$} & 14.4 & {$[14-14.9]$} & 0.116 & 13.8 & {$[13.0-14.5]$} & 14.3 & {$[14.0-14.6]$} & 0.205 & 14.1 & {$[13.6-14.5]$} & 14.3 & {$[13.9-14.7]$} & 0.409 & 14.2 & [13.8-14.6] & 14.2 & {$[13.7-14.7]$} & 0.861 \\
\hline Fat (g) & 58.9 & {$[56-61.8]$} & 58.6 & {$[55.9-61.3]$} & 0.891 & 58.4 & {$[53.6-63.1]$} & 58.8 & {$[56.6-61.0]$} & & 58.3 & {$[55.4-6$} & 59.0 & [56.4-61.7] & & & [56.3-61.5] & 58.5 & [55.4-61.6] & 0.862 \\
\hline Fat $(9$ & 28.2 & [26.9-2 & 28 & {$[27.2-29$} & 88 & 28.5 & {$[26.4-$} & & {$[27.3-2$} & & 28 & {$[2$} & 28.4 & [27.2-29.6] & & 28 & & 28.2 & {$[26.8$} & 0.851 \\
\hline & 246.6 & {$[238.1-2$} & 245.6 & [237.6-253.6] & 0.866 & 248.5 & [234.5-262.5] & 245.6 & {$[239.2-$} & & 248.7 & [240.1-257.3] & 243.9 & [236.2-251.7] & & 244.2 & 51.9] & 248.7 & 57.9] & 0.476 \\
\hline onergy) & 57.9 & [56.3-59.4] & 57.1 & [55.7-58.6] & 496 & 57.8 & {$[55.2-60.3]$} & 57.4 & & 0.819 & 57.7 & [56.2-59.3] & 57.3 & & & & & 7.6 & & 0.924 \\
\hline Fiber (g) & 12.0 & [11.4-12.7] & 12.4 & [11.8-13.0] & 0.420 & 12.5 & [11.4-13.6] & 12.2 & [11.7-12.7] & 0.614 & 12.1 & {$[11.5-12.8]$} & 12.3 & [11.7-12.9] & & & & & & \\
\hline Salt (g) & 8.4 & [7.8-9.0] & 9.2 & & 0.066 & 8.4 & {$[7.4-9.3]$} & 8.9 & {$[8.5-9.4]$} & 0.294 & 8.3 & {$[7.8-8.9]$} & 9.2 & {$[8.7-9.7]$} & 0.032 * & & {$[8.2-9.2]$} & 9.0 & & 0.594 \\
\hline Potassium (mg) & 2147 & [2053-2241] & 2176 & [2088-2264] & 0.663 & 2161 & [2006-2316] & 2163 & [2092-2233] & 0.988 & 2159 & [2064-2255] & 2165 & [2079-2251] & 0.932 & 2126 & [2042-2211] & 2213 & [2112-2315] & 0.208 \\
\hline Grain $(g)$ & 389.6 & [360.7-418.4] & 399.9 & [373.0-426.9] & 0.609 & 385.4 & [337.9-432.9] & 397.2 & {$[375.6-418.8]$} & 0.660 & 398.8 & [369.6-428.0] & 392.1 & [365.7-418.5] & 0.737 & 395.2 & [369.1-421.3] & 395.0 & [363.7-426.3] & 0.993 \\
\hline Potato (g) & 23.4 & {$[17.9-28.8]$} & 28.5 & {$[23.4-33.7]$} & 0.181 & 22.1 & [13-31.1] & 27.0 & {$[22.8-31.1]$} & 0.337 & 26.2 & [20.6-31.8] & 26.0 & [21.0-31.1] & 0.975 & 25.8 & {$[20.8-30.8]$} & 26.6 & [20.6-32.6] & 0.844 \\
\hline Vegetables (g) & 204.9 & {$[181.2-228.6]$} & 207.1 & [184.9-229.3] & 0.896 & 211.5 & {$[172.5-250.5]$} & 204.9 & {$[187.2-222.7]$} & 0.765 & 206.5 & {$[182.5-230.5]$} & 205.8 & [184.1-227.4] & 0.965 & 196.6 & {$[175.3-217.9]$} & 219.5 & [193.9-245] & 0.187 \\
\hline Green-yellow vegetables $(\mathrm{g})$ & 75.1 & [65.8-84.4] & 72.5 & [63.8-81.2] & 0.688 & 75.1 & [59.8-90.4] & 73.5 & {$[66.5-80.4]$} & 0.848 & 75.6 & [66.1-85.0] & 72.3 & {$[63.8-80.8]$} & 0.610 & 71.8 & [63.4-80.2] & 76.5 & {$[66.5-86.6]$} & 0.484 \\
\hline Other v & 129.8 & [113.6-145.9] & 134.6 & {$[119.5-149.7]$} & 0.673 & 136.4 & [109.8-162.9] & 131.5 & [119.4-143.6] & 0.743 & 130.9 & [114.6-147.3] & 133.5 & [118.7-148.3] & 0.819 & 124.8 & {$[110.4-139.3]$} & 142.9 & [125.6-160.3] & 0.125 \\
\hline Seaweec & 4.2 & {$[3.3-5.0]$} & 5.1 & {$[4.4-5.9]$} & 0.102 & 4.2 & {$[2.8-5.6]$} & 4.8 & {$[4.2-5.4]$} & 0.479 & 4.0 & {$[3.1-4.8]$} & 5.3 & {$[4.5-6.0]$} & 0.027 * & 4.3 & {$[3.6-5.1]$} & 5.2 & {$[4.3-6.1]$} & 0.140 \\
\hline y products $(\mathrm{g})$ & 54.3 & & 61.6 & & & 61.5 & {$[48.8-$} & 57.5 & {$[51.7-6$} & & 58 & {$[50.3-6$} & & {$[51.2-6$} & & & {$[53.1-$} & & {$[47.2-$} & 0.435 \\
\hline $\mathrm{Fish} / \mathrm{Pr}$ & 68.1 & $3.4-77$ & 79.9 & {$[70.9-8$} & & 62.7 & {$[46.9-7$} & & {$[69.7-$} & & 66.6 & {$[56.9$} & & {$[72.0-8-8-1-3$} & & 73.5 & {$[64.7-$} & 75.6 & {$[65.1-8$} & 0.760 \\
\hline cessed meat (g) & 78.0 & {$[67.7-88$} & 71.6 & {$[62.0-8$} & & 68.6 & {$[51.7-8$} & 75 & {$[68.2-$} & & 73.6 & & & {$[65.9-84$.} & 0.8 & 74.7 & {$[65.4$} & 74.4 & {$[63.2-$} & 0.965 \\
\hline & & {$[2$} & & & & & {$[2$} & & & & & & & & & & & & & 0.425 \\
\hline roducts & 119.1 & {$[98.5-13$} & 126.1 & {$[106.8-14$} & & 122.4 & & 122.9 & [107. & & & {$[109$.} & 116 & & & 127.3 & & 116.6 & & \\
\hline & & & 81.0 & & & & & & & & & & & & & & & & & \\
\hline & & & & & & & & & & & & & & & & & & & & \\
\hline & & & 105.1 & & & & & 107 & & & & & 116 & & & & & & & \\
\hline $\mathrm{O}$ & 76.6 & & 53.0 & & & 83 & & 59 & & & 82 & & 48 & & & & & & & \\
\hline & 6.3 & & 7.2 & & & 6.5 & & 6. & & & 6. & & 7.4 & & & 6.6 & & & & \\
\hline & 2.2 & & 2.4 & & & 2.2 & & 2.3 & & 0.8 & 2.2 & & 2.4 & & 0.6 & 2.1 & & 2.6 & & \\
\hline Oil (g) & 11.9 & [10.3-13.4] & 12.1 & {$[10.6-13.5]$} & 0.846 & 11.5 & {$[9.0-14.0]$} & 12.1 & [10.9-13.2] & 0.694 & 11.6 & {$[10-13.1]$} & 12.3 & [10.9-13.7] & 0.484 & 12.0 & [10.6-13.4] & 11.9 & {$[10.2-13.6]$} & 0.919 \\
\hline Seasonings (g) & 23.7 & {$[21.2-26.2]$} & 26.6 & {$[24.3-29.0]$} & 0.097 & 22.8 & {$[18.7-27.0]$} & 25.8 & {$[23.9-27.6]$} & 0.210 & 23.9 & [21.4-26.5] & 26.3 & {$[24-28.6]$} & 0.174 & 25.7 & {$[23.4-28]$} & 24.6 & {$[21.8-27.3]$} & 0.545 \\
\hline
\end{tabular}

${ }^{*} p<0.05$. Energy intake was adjusted for age, sex, duration of diabetes, BMI, HbA1c, using OHA, and using insulin. Nutrients and food group intakes were adjusted for age, sex, duration of diabetes, BMI, HbA1c, using OHA, using insulin, and energy intake per day. BMI, body mass index; OHA, oral hypoglycemic agent. 
Table 4. Dietary intake and response to the Diabetes Family Behavior Checklist scale for medical nutritional therapy in women with type 2 diabetes $(N=120)$.

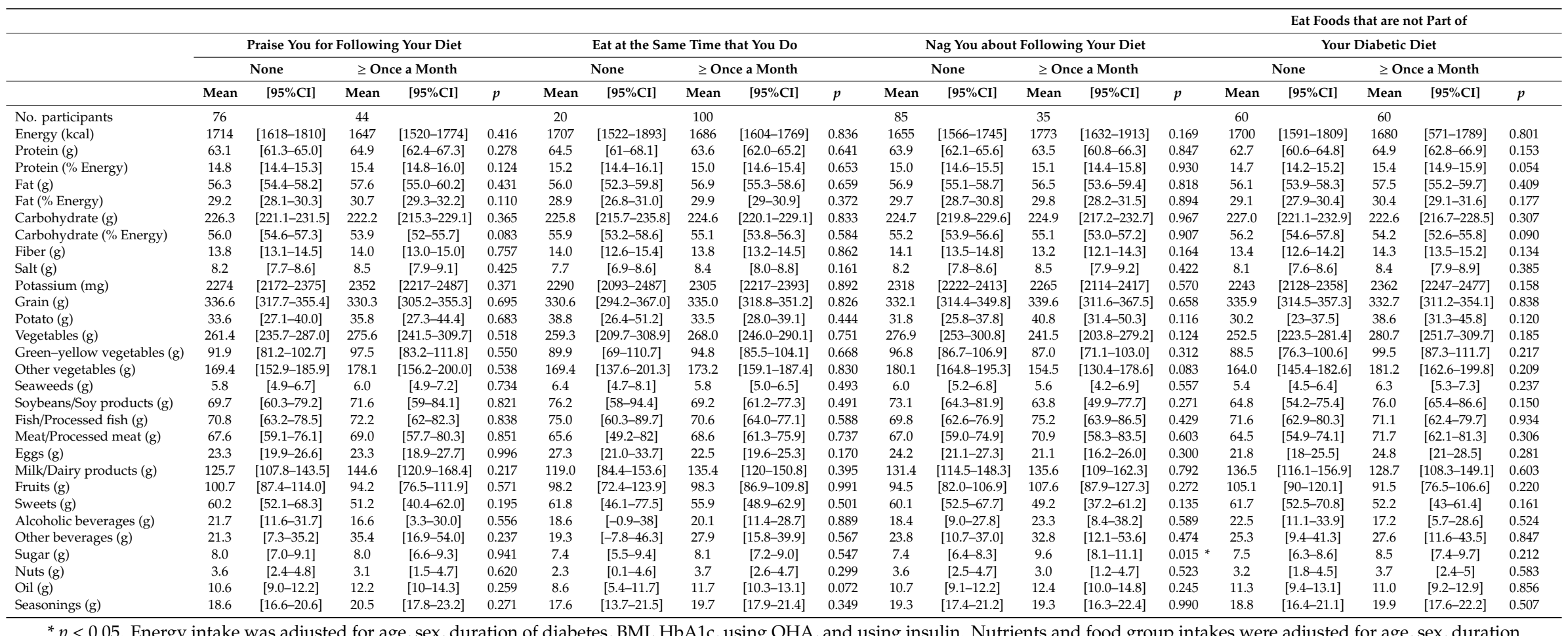

of diabetes, BMI, HaA1c, using $\mathrm{OHA}$, using insulin, and energy intake per day. BMI, body mass index; OHA, oral hypoglycemic agent. 


\section{Discussion}

The current study determined the association between habitual dietary intake by food group and nutrient levels among Japanese with type 2 diabetes and types of daily family support for MNT using DFBC.

The present results suggest that family members regularly discussing MNT with patients with type 2 diabetes by such means as "praise for following your diet" or "nag about not following your diet" was related to patients' intake of desirable food groups in comparison with other cases in which such conversations did not take place, though there was no significant difference in energy intake. Patients with type 2 diabetes need family support and assistance in self-management practices through help with strategic planning, goal setting, and problem-solving [29]. Patient-family relationships have been shown to improve adherence to general dietary recommendations and motivation for diabetes treatment $[13,19,29]$. Although it is considered important in MNT for diabetes to provide and put into practice specific indications, increases in patients' self-efficacy including a positive attitude toward treatment can arise from positive support such as praise and encouragement [30,31]. Our results showed that those responding positively to "nag about not following your diet" had a higher intake of fish and shellfish than those who responded "none." To maintain this result over the long term, it would be necessary for family members to encourage patients to increase fish and shellfish intake while "praising the patient for following their diet."

In addition, the intake of salt was significantly higher in study participants selecting the response indicating that they experienced nagging about not following their diet. The amount of salt intake is difficult to grasp visually as salt is found in seasonings and processed foods [32,33]. It was reported that seasonings contributed to about $60 \%$ of the salt intake in Japanese people [34]. In MNT for type 2 diabetes, there is a need to educate patients and their family members on how to reduce salt intake such as the characteristics of food with a high-salt content, how to read nutritional information on labels, and how to reduce salt in cooking [34-36]. This information is important when educating patients on adequate intake of energy and certain food groups.

As for "eat at the same time that you do," patients who indicated that this behavior occurred once a month or more had a higher energy intake compared to those with a response of "none," but no significant difference was seen in intakes of food groups and nutrients. This indicates that family members of patients with type 2 diabetes may not have a good understanding of what food groups and how much food intake are effective for MNT when eating with the patient. An international joint research project reported that a low percentage of family members of people with diabetes know how to help persons with their diabetes (37.1\%) and participate in diabetes educational programs $(23.1 \%)$, although most of those who did participate found such programs helpful (72.1\%) [37]. A family member should be present at the time of the MNT education to share information on dietary intake in diabetes care and closely support a patient's MNT. Such participation will enhance the patient's self-efficacy, including that related to MNT, encourage a positive attitude toward treatment, and increase the consumption of healthy foods such as vegetables and fruit $[12,13]$.

Patients indicating that they "eat foods that are not part of their diabetes diet" had higher dietary fiber and vegetable intake than those who did not. High dietary fiber intake including that of vegetables is also important for family members without diabetes because MNT for diabetes is also helpful in the prevention of many lifestyle-related diseases, and not only diabetes [38-40]. By having both patients with type 2 diabetes and their family members consuming recommended daily values for vegetables and dietary fiber (fiber intake: $\geq 14 \mathrm{~g} / 1000 \mathrm{kcal}$ by the American Diabetes Association [41] and $\geq 20 \mathrm{~g} /$ day by the Japan Diabetes Society [42]), the whole family would benefit by avoiding lifestyle-related diseases and have a higher quality of life.

When subgroup analysis by sex was performed, the results for male patients roughly followed the same pattern for significant differences as in the overall study population. Some additional relationships, such as higher fiber intake, higher seaweed intake, and fewer preferred beverages other than alcohol were shown for negative feedback such as "being nagged about not following diet" and 
"eating foods that are not part of the diabetes diet." On the other hand, for female patients, significant differences were attenuated in the areas where significant differences were found in the overall study population. This suggests that familial dietary support is associated with preferred habitual dietary intake, but this tendency was especially strong for male patients and a reverse association was found in female patients.

The reason why support for MNT by family members was significantly associated with good habitual dietary intake in males but not in females may be because the attributes of those providing the support were different. According to Table 1, most male patients $(87.6 \%)$ received support from their wives while $65.8 \%$ of females received support from husbands and $16.6 \%$ from children.

In daily eating, food and nutritional knowledge regarding the composition of healthy meals greatly affects the content of the meals and varies according to sex. Women tend to desire knowledge about food and nutrients to a greater degree than men [43,44]. This knowledge can result in eating a diet rich in fruits and vegetables and low in fats $[43,45]$. Furthermore, it has been reported that $76 \%$ of women are in charge of shopping for groceries, and $91 \%$ of women are mainly involved in preparing meals in Japanese families [46]. In addition, it was reported that knowledge of food and nutrients as well as practical skills in food selection and preparation are usually at a low level in young age groups [44,45].

In summary, male patients are most likely to receive support regarding knowledge of food and nutrients from their wives who have knowledge of nutrition and practical skills in food preparation and female patients receive support from husbands and children who have less knowledge of food. Also, most female patients are in charge of preparing daily meals. Hence, it can be expected that female patients are likely to work on MNT on their own, and that family support would not lead to dietary improvements. In order to prevent disparities in the support situation due to sex differences, care should be taken to ensure that family support for female patients is effectively linked to MNT by involving family members, so that they may acquire basic food knowledge and cooking skills.

Limitations of this study must be considered. First, the potential for bias, such as measurement errors in dietary assessments, confounding factors, and informative censoring, cannot be ruled out entirely although this study is adjusted for age, sex, duration of diabetes, BMI, HbA1c, using OHA, and using insulin as confounders for dietary intake using multiple regression analysis. Second, as this is a cross-sectional study, cause and effect relationships could not be concluded. Further cohort studies and randomized trials are needed for clarify whether family support encouraging MNT would affect habitual dietary intake in clinical practice. Third, although $81.6 \%$ of patients with type 2 diabetes completed our survey, the potential effect of a partial non-response bias should be addressed. It is known that people who participate in research studies tend to have health awareness and be well motivated compared with those who do not $[47,48]$. Finally, our results may not be applicable to populations with different lifestyles or genetic factors. For example, the Japanese patients with type 2 diabetes were shown to consume a high-carbohydrate low-fat diet compared with Western patients with diabetes [49], and their mean BMI was low compared to persons from Western countries [50]. It is also known that racial/ethnic variations differ among family household compositions and family dynamics [17]. Considering racial/ethnic-specific characteristics and large intercultural differences is important in exploring effective MNT. Further research on this topic is needed.

\section{Conclusions}

In conclusion, we clarified in detail the relationships between the type of family support received by patients with type 2 diabetes in daily life and their dietary intake by food group and nutrient level. We showed that support required for medical treatment, which involves not only negative feedback but also positive feedback among family support, can be linked to desirable dietary intake for diabetes care, and that it is necessary to pay particular attention to family support for women. Further longitudinal studies or interventional studies are needed for tracking the dietary intake of patients with type 2 
diabetes, and exploring family support in consideration of sex difference is important for the more effective practice of MNT.

Author Contributions: Conceptualization: C.H., K.F., and H.S.; data curation: C.H., M.H., S.Y.M., Y.T., M.T., K.F., N.K., H.Y., Y.K., K.I., S.T., H.M., and H.S.; formal analysis: C.H. and H.S.; funding acquisition: C.H. and H.S.; investigation: C.H., M.H., S.Y.M., Y.T., M.T., K.F., N.K., H.Y., Y.K., K.I., S.T., H.M, and H.S.; methodology: C.H., M.H., S.Y.M., Y.T., S.T., and H.S.; project administration: H.S.; resources: N.K., H.Y., Y.K., K.I., H.M., and H.S.; software: C.H. and H.S.; supervision: H.S.; validation: C.H. and H.S.; visualization: C.H. and H.S.; writing-original draft: C.H., K.F., and H.S.; writing-review and editing: C.H., M.H., S.Y.M., Y.T., M.T., K.F., N.K., H.Y., Y.K., K.I., S.T., H.M., and H.S. H.S. is the guarantor of this work and, as such, had full access to all of the data in the study and takes responsibility for the integrity of the data and the accuracy of the data analysis. All authors have read and agreed to the published version of the manuscript.

Funding: The research was funded by Grants for young researchers from Japan Association for Diabetes Education and Care. H.S. was support by a Grant-in-Aid for Scientific Research (\#19H04028) from the Japan Society for the Promotion of Science (JSPS). The sponsor had no role in the design and conduct of the study; collection, management, analysis, and interpretation of the data; or preparation, review, or approval of the manuscript.

Acknowledgments: The authors thank the participants who took part in the study. We also thank Mami Haga and Natsuko Tada, Niigata University, for their excellent secretarial assistance.

Conflicts of Interest: The authors declare no conflict of interest. The funders had no role in the design of the study; in the collection, analyses, or interpretation of data; in the writing of the manuscript; or in the decision to publish the results.

\section{References}

1. Norris, S.L.; Lau, J.; Smith, S.J.; Schmid, C.H.; Engelgau, M.M. Self-management education for adults with type 2 diabetes: A meta-analysis of the effect on glycemic control. Diabetes Care 2002, 25, 1159-1171. [CrossRef] [PubMed]

2. Rothman, R.L.; Malone, R.; Bryant, B.; Shintani, A.K.; Crigler, B.; Dewalt, D.A.; Dittus, R.S.; Weinberger, M.; Pignone, M.P. A randomized trial of a primary care-based disease management program to improve cardiovascular risk factors and glycated hemoglobin levels in patients with diabetes. Am. J. Med. 2005, 118, 276-284. [CrossRef] [PubMed]

3. Home, P.; Mant, J.; Diaz, J.; Turner, C. Guideline Development Group. Management of type 2 diabetes: Summary of updated NICE guidance. BMJ 2008, 336, 1306-1308. [CrossRef] [PubMed]

4. He, X.; Li, J.; Wang, B.; Yao, Q.; Li, L.; Song, R.; Shi, X.; Zhang, J. Diabetes self-management education reduces risk of all-cause mortality in type 2 diabetes patients: A systematic review and meta-analysis. Endocr. 2017, 55, 712-731. [CrossRef] [PubMed]

5. Kravitz, R.L.; Hays, R.D.; Sherbourne, C.D.; DiMatteo, M.R.; Rogers, W.H.; Ordway, L.; Greenfield, S. Recall of recommendations and adherence to advice among patients with chronic medical conditions. Arch. Intern. Med. 1993, 153, 1869-1878. [CrossRef]

6. Alan, M. Improving Patient Adherence. Clin. Diabetes 2006, 24, 71-77.

7. Cohen, S. Psychosocial models of the role of social support in the etiology of physical disease. Health Psychol. 1988, 7, 269-297. [CrossRef]

8. Frosch, D.L.; Uy, V.; Ochoa, S.; Mangione, C.M. Evaluation of a behavior support intervention for patients with poorly controlled diabetes. Arch. Intern. Med. 2011, 171, 2011-2017. [CrossRef]

9. Baum, A.; Revenson, T.A.; Singer, J.E. Handbook of Health Psychology; Lawrence Erlbaum: Hillsdale, NJ, USA, 2000.

10. American Diabetes Association. 5. Facilitating Behavior Change and Well-being to Improve Health Outcomes: Standards of Medical Care in Diabetes-2020. Diabetes Care 2020, 43, 48-65. [CrossRef]

11. Kodama, S.; Morikawa, S.; Horikawa, C.; Ishii, D.; Fujihara, K.; Yamamoto, M.; Osawa, T.; Kitazawa, M.; Yamada, T.; Kato, K.; et al. Effect of family-oriented diabetes programs on glycemic control: A meta-analysis. Fam. Pract. 2019, 36, 387-394. [CrossRef]

12. Wichit, N.; Mnatzaganian, G.; Courtney, M.; Schulz, P.; Johnson, M. Randomized controlled trial of a family-oriented self-management program to improve self-efficacy, glycemic control and quality of life among Thai individuals with Type 2 diabetes. Diabetes Res. Clin. Pract. 2017, 123, 37-48. [CrossRef] [PubMed] 
13. Baig, A.A.; Benitez, A.; Quinn, M.T.; Burnet, D.L. Family interventions to improve diabetes outcomes for adults. Ann. N. Y. Acad. Sci. 2015, 1353, 89-112. [CrossRef] [PubMed]

14. Choi, S.E. Diet-specific family support and glucose control among Korean immigrants with type 2 diabetes. Diabetes Educ. 2009, 35, 978-985. [CrossRef] [PubMed]

15. Watanabe, K.; Kurose, T.; Kitatani, N.; Yabe, D.; Hishizawa, M.; Hyo, T.; Seino, Y. The role of family nutritional support in Japanese patients with type 2 diabetes mellitus. Intern. Med. 2010, 49, 983-989. [CrossRef]

16. Mayberry, L.S.; Osborn, C.Y. Family support, medication adherence, and glycemic control among adults with type 2 diabetes. Diabetes Care 2012, 35, 1239-1245. [CrossRef]

17. Bennich, B.B.; Røder, M.E.; Overgaard, D.; Egerod, I.; Munch, L.; Knop, F.K.; Vilsbøll, T.; Konradsen, H. Supportive and non-supportive interactions in families with a type 2 diabetes patient: An integrative review. Diabetol. Metab. Syndr. 2017, 9, 57. [CrossRef]

18. Yun, J.; Kim, K. Relationships of family support, diet therapy practice and blood glucose control in typeII diabetic patients. Nutrition Res. Pract. 2009, 3, 141-148. [CrossRef]

19. Mayberry, L.S.; Osborn, C.Y. Family involvement is helpful and harmful to patients' self-care and glycemic control. Patient Educ. Couns. 2014, 97, 418-425. [CrossRef]

20. Kobayashi, M.; Yamazaki, K.; Hirao, K.; Oishi, M.; Kanatsuka, A.; Yamauchi, M.; Takagi, H.; Kawai, K. Japan Diabetes Clinical Data Management Study Group. The status of diabetes control and antidiabetic drug therapy in Japan - a cross-sectional survey of 17,000 patients with diabetes mellitus (JDDM 1). Diabetes Res. Clin. Pract. 2006, 73, 198-204. [CrossRef]

21. Oishi, M.; Yamazaki, K.; Okuguchi, F.; Sugimoto, H.; Kanatsuka, A.; Kashiwagi, A. Japan Diabetes Clinical Data Management Study Group. Changes in oral antidiabetic prescriptions and improved glycemic control during the years 2002-2011 in Japan (JDDM32). J. Diabetes Investig. 2014, 5, 581-587. [CrossRef]

22. Schafer, L.C.; McCaul, K.D.; Glasgow, R.E. Supportive and nonsupportive family behaviors: Relationships to adherence and metabolic control in persons with type I diabetes. Diabetes Care 1986, 9, 179-185. [CrossRef] [PubMed]

23. Hara, Y.; Iwashita, S.; Ishii, K.; Inada, C.; Okada, A.; Tajiri, Y.; Nakayama, H.; Kato, T.; Nishida, K.; Ogata, Y.; et al. The reliability and validity of the Japanese version of the Diabetes Family Behavior Checklist (DFBC) for assessing the relationship between type 2 diabetes mellitus patients and their families with respect to adherence to treatment regimen. Diabetes Res. Clin. Pract. 2013, 99, 39-47. [CrossRef] [PubMed]

24. Takahashi, K.; Yoshimura, Y.; Kaimoto, T.; Kunii, D.; Komatsu, T.; Yamamoto, S. Validation of a food frequency questionnaire based on food groups for estimating individual nutrient intake. Jpn. J. Nutr. 2001, 59, 221-232. [CrossRef]

25. Glasgow, R.E.; Toobert, D.J. Social environment and regimen adherence among type II diabetic patients. Diabetes Care 1988, 11, 377-386. [CrossRef]

26. Wen, L.K.; Shepherd, M.D.; Parchman, M.L. Family support, diet, and exercise among older Mexican Americans with Type 2 diabetes. Diabetes Educ. 2004, 30, 980-993. [CrossRef]

27. Ministry of Education, Culture, Sports, Science and Technology, Japan. STANDARD TABLES OF FOOD COMPOSITION IN JAPAN—2015—(Seventh Revised Version). Available online: http://www.mext.go.jp/en/ policy/science_technology/policy/title01/detail01/1374030.htm (accessed on 11 August 2020).

28. Sone, H.; Tanaka, S.; Iimuro, S.; Tanaka, S.; Oida, K.; Yamasaki, Y.; Oikawa, S.; Ishibashi, S.; Katayama, S.; Yamashita, H.; et al. Long-term lifestyle intervention lowers the incidence of stroke in Japanese patients with type 2 diabetes: A nationwide multicentre randomised controlled trial (the Japan Diabetes Complications Study). Diabetologia 2010, 53, 419-428. [CrossRef]

29. Keogh, K.M.; Smith, S.M.; White, P.; McGilloway, S.; Kelly, A.; Gibney, J.; O’Dowd, T. Psychological family intervention for poorly controlled type 2 diabetes. Am. J. Manag. Care 2011, 17, 105-113.

30. Bandura, A. Self-efficacy: Toward a Unifing Theory of Behavioral Change. Psychol. Rev. 1977, 84, $191-215$. [CrossRef]

31. Bandura, A. Self-Efficacy in Changing Societies; Cambridge University Press: Cambridge, UK, 1995.

32. Magriplis, E.; Farajian, P.; Pounis, G.D.; Risvas, G.; Panagiotakos, D.B.; Zampelas, A. High sodium intake of children through 'hidden' food sources and its association with the Mediterranean diet: The GRECO study. J. Hypertens. 2011, 29, 1069-1076. [CrossRef] 
33. Kastorini, C.M.; Milionis, H.J.; Kalantzi, K.; Trichia, E.; Nikolaou, V.; Vemmos, K.N.; Goudevenos, J.A.; Panagiotakos, D.B. The mediating effect of the Mediterranean diet on the role of discretionary and hidden salt intake regarding non-fatal acute coronary syndrome or stroke events: Case/case-control study. Atherosclerosis 2012, 225, 187-193. [CrossRef]

34. Asakura, K.; Uechi, K.; Masayasu, S.; Sasaki, S. Sodium sources in the Japanese diet: Difference between generations and sexes. Public Health Nutr. 2016, 19, 2011-2023. [CrossRef] [PubMed]

35. Horikawa, C.; Murayama, N.; Sasaya, M.; Sakai, M.; Sano, M.; Takizawa, N.; Takano, M. Prediction of high salt and low potassium intake behavior from urinary sodium and potassium excretion in Japan. Progr. Nutr. 2019, 21, 149-163.

36. Wyness, L.A.; Butriss, J.L.; Stanner, S.A. Reducing the population's sodium intake: The UK Food Standards Agency's salt reduction programme. Public Health Nutr. 2012, 15, 254-261. [CrossRef] [PubMed]

37. Kovacs, B.K.; Nicolucci, A.; Holt, R.I.G.; Willaing, I.; Hermanns, N.; Kalra, S.; Wens, J.; Pouwer, F.; Skovlund, S.E.; Peyrot, M.; et al. Diabetes Attitudes, Wishes and Needs second study (DAWN2 ${ }^{\mathrm{TM}}$ ): Cross-national benchmarking indicators for family members living with people with diabetes. Diabet. Med. 2013, 30, 778-788. [CrossRef] [PubMed]

38. Tanaka, S.; Yoshimura, Y.; Kamada, C.; Tanaka, S.; Horikawa, C.; Okumura, R.; Ito, H.; Ohashi, Y.; Akanuma, Y.; Yamada, N.; et al. Intakes of dietary fiber, vegetables, and fruits and incidence of cardiovascular disease in Japanese patients with type 2 diabetes. Diabetes Care 2013, 36, 3916-3922. [CrossRef]

39. Wang, P.Y.; Fang, J.C.; Gao, Z.H.; Zhang, C.; Xie, S. Higher intake of fruits, vegetables or their fiber reduces the risk of type 2 diabetes: A meta-analysis. J. Diabetes. Investig. 2016, 7, 56-69. [CrossRef]

40. Reynolds, A.; Mann, J.; Cummings, J.; Winter, N.; Mete, E.; Morenga, L.T. Carbohydrate quality and human health: A series of systematic reviews and meta-analyses. Lancet 2019, 393, 434-445. [CrossRef]

41. Evert, A.B.; Dennison, M.; Gardner, C.D.; Garvey, W.T.; Lau, K.H.K.; MacLeod, J.; Mitri, J.; Pereira, R.F.; Rawlings, K.; Robinson, S.; et al. Nutrition Therapy for Adults With Diabetes or Prediabetes: A Consensus Report. Diabetes Care 2019, 42, 731-754. [CrossRef]

42. Japan Diabetes Society. Treatment Guide for Diabetes 2016-2017; Bunkodo: Tokyo, Japan, 2016.

43. Wardle, J.; Parmenter, K.; Waller, J. Nutrition knowledge and food intake. Appetite 2000, 34, $269-275$. [CrossRef]

44. Hendrie, G.A.; Coveney, J.; Cox, D. Exploring nutrition knowledge and the demographic variation in knowledge levels in an Australian community sample. Public Health Nutr. 2008, 11, 1365-1371. [CrossRef]

45. Spronk, I.; Kullen, C.; Burdon, C.; O'Connor, H. Relationship between nutrition knowledge and dietary intake. Br. J. Nutr. 2014, 111, 1713-1726. [CrossRef] [PubMed]

46. International Social Survey Programme. ZA5900: International Social Survey Programme: Family and Changing Gender Roles IV-ISSP 2012. Available online: https://dbk.gesis.org/dbksearch/sdesc2.asp?db=e\& no $=5900$ (accessed on 11 August 2020).

47. Svensson, T.; Inoue, M.; Sawada, N.; Iwasaki, M.; Sasazuki, S.; Shimazu, T.; Yamaji, T.; Ikeda, A.; Kawamura, N.; Mimura, M.; et al. The association between complete and partial non-response to psychosocial questions and suicide: The JPHC Study. Eur. J. Public Health 2015, 25, 424-430. [CrossRef] [PubMed]

48. Kypri, K.; Samaranayaka, A.; Connor, J.; Langley, J.D.; Maclennan, B. Non-response bias in a web-based health behaviour survey of New Zealand tertiary students. Prev. Med. 2011, 53, 274-277. [CrossRef] [PubMed]

49. Horikawa, C.; Yoshimura, Y.; Kamada, C.; Tanaka, S.; Tanaka, S.; Takahashi, A.; Hanyu, O.; Araki, A.; Ito, H.; Tanaka, A.; et al. Dietary intake in Japanese patients with type 2 diabetes: Analysis from Japan Diabetes Complications Study. J. Diabetes Invest. 2014, 5, 176-187. [CrossRef] [PubMed]

50. Sone, H.; Ito, H.; Ohashi, Y.; Akanuma, Y.; Yamada, N. Japan Diabetes Complication Study Group. Obesity and type 2 diabetes in Japanese patients. Lancet 2003, 361, 85. [CrossRef]

(C) 2020 by the authors. Licensee MDPI, Basel, Switzerland. This article is an open access article distributed under the terms and conditions of the Creative Commons Attribution (CC BY) license (http://creativecommons.org/licenses/by/4.0/). 